\title{
Encapsulation of peptides in biodegradable microspheres prolongs their MHC class-I presentation by dendritic cells and macrophages in vitro
}

\author{
Régine Audran $^{\mathrm{a}, 1}$, Katrin Peter ${ }^{\mathrm{a}, 1}$, Jens Dannull ${ }^{\mathrm{b}}$, Ying Men ${ }^{\mathrm{c}}$, Elke Scandella ${ }^{\mathrm{b}}$, \\ Marcus Groettrup ${ }^{\mathrm{b}}$, Bruno Gander ${ }^{\mathrm{c}}$, Giampietro Corradin ${ }^{\mathrm{a}, *}$ \\ a Institute of Biochemistry, University of Lausanne, Chemin des Boveresses 155, 1066 Epalinges, Switzerland \\ b Research Department, Cantonal Hospital St. Gallen, St. Gallen, Switzerland \\ c Institute of Pharmaceutical Sciences, ETHZ, Zürich, Switzerland
}

\begin{abstract}
Biodegradable microspheres (MS) consisting of poly(D,L-lactide-co-glycolide) (PLGA) represent a promising alternative to conventional adjuvants. The adjustable pulsatile release of encapsulated material from such MS offers the potential to mimic the priming and boosting injections of conventional immunization regimens. In this paper, we demonstrate that MS can serve as antigen reservoirs in antigen presenting cells (APC), so that antigen is presented for extended periods of time (up to 9 days). In particular, we could show by measurement of IFN- $\gamma$ production that encapsulated peptides were presented to cytotoxic T lymphocytes (CTL) by mouse and human macrophages as well as by human dendritic cells in vitro for a longer time period as compared to soluble peptides. The extended antigen presentation may thus improve the CTL response in vivo. These results may be of paramount importance in cancer vaccination therapy since MS may serve as antigen reservoirs to extend the presentation time by APC used to boost the patient's immune response to tumor antigens.
\end{abstract}

Keywords: Biodegradable microspheres; Poly (D,L-lactide-co-glycolide); CTL peptides; Dendritic cells; Macrophages

\section{Introduction}

Biodegradable microspheres (MS) prepared from poly(D,L-lactide-co-glycolide) (PLGA) and poly(D,L-lactide) (PLA) have been used for quite some time as injectable drug delivery systems. The adjustable degradation rate of these biodegradable MS provides an efficient means to control and prolong the release of encapsulated compounds [1]. Therefore, PLGA/PLA MS are of particular interest as technology platforms for new vaccines, as they offer prolonged and pulsatile release of encapsulated antigens. The prolonged and pulsatile release of antigen from MS may mimic the priming and boosting injections of conventional immunization schedules. It has indeed been shown in numerous examples, that a sustained, robust immune response is achieved with antigens microencapsulated in biodegradable MS [2-8]. Therefore, the use of MS represents a

\footnotetext{
* Corresponding author. Tel.: +41-21-692-5731; fax: +41-21-692-5705

E-mail address: giampietro.corradin@ib.unil.ch (G. Corradin).

1 Both authors contributed equally to this work.
}

very attractive approach for vaccine development because it would reduce the number of injections a person will be subjected to and would increase vaccine coverage in countries where the dropout rate for successive immunizations is high.

A further possible exploitation of the characteristics of biodegradable MS is their use as antigen reservoirs in antigen presenting cells (APC), such as to furnish antigen for presentation for an extended period of time. This strategy appears particularly attractive in cancer vaccination therapy in which APC are pulsed with antigen to boost the patient's immune response to tumors [9]. It can be predicted that while pulsing of APC with soluble antigen would lead to a limited temporal presentation of the MHC-peptide complex due to its intrinsic dissociation rate and turnover, the MS pulsed APC could benefit from a longer antigen presence and a constant re-furnishment of MHC complexes. We show in this report that long persistence of antigen presentation is, in fact, achieved when professional APC are loaded with cytotoxic T lymphocyte (CTL) epitopes encapsulated in MS. 


\section{Materials and methods}

\subsection{Antigens}

Three synthetic CTL epitopes were used, the $\mathrm{H}-2 \mathrm{~K}^{\mathrm{d}}$ restricted peptide (SYIPSAEKI) derived from the circumsporozoite (CS) protein of Plasmodium berghei, $\mathrm{PbCS}_{245-253}(\mathrm{~Pb})$ [10], the HLA A2.01 restricted peptide (YLNKIQNSL) derived from the CS protein of Plasmodium falciparum, strain NF54, PfCS $334-342$ (Pf) [11], and the HLA A2.01 restricted peptide (GILGFVFTL) derived from the influenza matrix protein, $\mathrm{MP}_{58-66}$ [12] (Flu).

\subsection{Antigen microencapsulation and microspheres}

The peptides $\mathrm{Pb}$, Pf, and Flu were microencapsulated in PLGA 50:50 (Resomer RG502, Boehringer Ingelheim, D) by spray-drying as previously described [1]. The peptide loading of the resulting MS (MS-Pb, MS-Pf, and MS-Flu) was 2, 4.8, and $3.2 \mu \mathrm{g} / \mathrm{mg} \mathrm{MS}$, respectively. Their diameter varied between 1 and $10 \mu \mathrm{m}$. These antigen loaded MS are capable of releasing their contents in a pulsatile pattern [1]. Empty MS (MSe) were prepared using the same polymer.

\subsection{Murine APC}

Murine RAW macrophages (cell line 264) were grown in complete Dulbecco's modified Eagle's medium (cDMEM): DMEM (GIBCO BRL, Life Technologies Inc., Paisley, United Kingdom), 5\% heat inactivated fetal calf serum (FCS, Seromed ${ }^{\circledR}$ Biochrom KG, Berlin, Germany), 2 mM L-glutamine, and $10 \mathrm{mM}$ HEPES. Bone marrow-derived macrophages (BMM) were prepared from BALB/c mouse tibias and femurs as described elsewhere [13]. Briefly, medulla cells from two legs were cultivated 9-12 days on low adherence Petri dishes (Biocon, Dortmund, Germany) in $100 \mathrm{ml}$ of cytokine enriched medium: 50\% cDMEM, 20\% horse serum, 30\% L929 conditioned medium. After this time, floating cells were removed. Adherent cells were detached by cell scraping after $15 \mathrm{~min}$ of incubation at $4{ }^{\circ} \mathrm{C}$ and washed. Cells were seeded at $5 \times 10^{5}$ cells per well in 24 well plates for $6 \mathrm{~h}$ permitting cell spreading prior to the peptide presentation assay.

\subsection{Human APC}

\subsubsection{Macrophages}

PBMC were prepared from fresh blood by Ficoll-Paque (Pharmacia LKB, Uppsala, Sweden) density centrifugation and cultivated 7 days in a Petri dish (Biocon) at $10^{6}$ cells $/ \mathrm{ml}$ in DMEM, 10\% human AB serum, $10 \mathrm{mM}$ HEPES, $2 \mathrm{mM}$ L-glutamine, $1 \mathrm{mM}$ sodium pyruvate, and $1 \%$ of a mixture of non-essential amino acids. Non-adherent cells were removed and remaining cells detached with a plunger after incubation for $15 \mathrm{~min}$ at $4{ }^{\circ} \mathrm{C}$. Cells were seeded at $6 \times 10^{4}$ cells per well in 48 well plates and cultured for $4 \mathrm{~h}$ prior to peptide presentation assay.

\subsubsection{Dendritic cells}

Monocytes were isolated from fresh blood by Ficoll-Paque density centrifugation and $\mathrm{CD}_{1}{ }^{+}$magnetic sorting and cultured for 5 days in GM-CSF (50 ng/ml, Leukomax, Roche, Basel, Switzerland) and IL-4 (1000 U/ml, R\&D Systems, Abingdon, United Kingdom) for differentiation to immature DCs as previously described [14]. DCs were seeded at $10^{6}$ cells per well in 24 well plates prior to peptide presentation assay.

\subsection{CTL}

The murine PbCS.C7 CD8 ${ }^{+}$CTL clone specific for the $\mathrm{Pb}$ epitope was cultured in cDMEM supplemented with $30 \mathrm{IU} / \mathrm{ml} \mathrm{IL}-2$ (EL-4 cell culture supernatant) and $50 \mu \mathrm{M}$ 2-mercaptoethanol and was periodically re-stimulated with peptide pulsed APC as previously described [10]. Human CTL clones, specific for Flu (kindly provided by Dr. Danila Valmori) or Pf were cultured in 96-well round bottom plates in Iscove's Modified Dulbecco's Medium supplemented with 10\% human AB serum (Blutspendedienst SRK, Bern, Switzerland), $10 \mathrm{mM}$ HEPES, $1 \mathrm{mM}$ sodium pyruvate, $1 \%$ non-essential amino acids, $1 \%$ penicilline/streptomycin (GIBCO BRL Life Technologies, Paisley, UK), $10 \mu \mathrm{g} / \mathrm{ml}$ Ciproxin (Bayer AG, Leverkusen, Germany), and $150 \mathrm{IU} / \mathrm{ml}$ hu-IL2 (GlaxoSmithKline, Thoerishaus, Switzerland). CTLs were periodically re-stimulated (every 3-4 weeks) with $1 \mu \mathrm{g} / \mathrm{ml}$ phytohemagglutinin (Sigma) in presence of irradiated allogeneic PBMC. These three $\mathrm{CD}^{+}$clones produce IFN- $\gamma$ upon activation.

\subsection{Kinetics of antigen presentation}

In all cases, APC were cultured overnight in the presence of $1 \mu \mathrm{M}$ soluble or encapsulated peptide in a volume adapted to the well size and allowing optimal phagocytosis of the MS ( $0.5 \mathrm{ml}$ for the RAW cell line and murine macrophages in 24 well plates, $170 \mu \mathrm{l}$ for human macrophages in 48 well plates, and $1 \mathrm{ml}$ for DCs in 24 well plates). Free MS were eliminated from the macrophage monolayer by extensive washing. To assure that the antigen presentation kinetics observed with antigen-loaded MS was not due to peptide release from MS remaining free in culture after washing, we confirmed by visual inspection that the washed cultures did not reveal free MS in the culture medium. This was accomplished by culturing cells in large wells to facilitate washing and at a cell density allowing the formation of monolayers and optimal MS uptake. Moreover, the periphery of culture wells was systematically cleared from free MS by aspiration. Given that a 10 to 100 -fold decrease in antigen concentration drastically diminished or abolished antigen recognition by CTLs (data not shown) it seems 
safe to assume that residual MS that might have remained in culture medium after washing can not account for the prolonged antigen presentation observed for MS.

In addition, RAW cells were irradiated before the last wash to inhibit their growth. Since DCs are not adherent, they were separated from non-phagocytosed MS by labeling them with a mouse anti-CD44 antibody (clone B-F24, Diaclone, Besançon, France) plus anti-mouse Ig magnetic beads (Goat anti-mouse Microbeads) followed by the passage through a magnetic column (Minimacs, Miltenyi, Bergish Gladbach, Germany). DCs were then seeded in round bottom well plates (about $3 \times 10^{4}$ DCs per well).
CTLs were added (1 million/ml for murine cultures, 1000 and 4000 CTLs in $150 \mu$ l to human macrophage and DC cultures, respectively) at various time points. Co-culture supernatants were collected after $24 \mathrm{~h}$ and stored at $-20^{\circ} \mathrm{C}$ before IFN- $\gamma$ evaluation.

\subsection{Measurement of IFN- $\gamma$ production}

Murine IFN- $\gamma$ was determined as described by Migliorini et al. [15] and human IFN- $\gamma$ by the human IFN- $\gamma$ ELISA kit (Mabtech, Stockholm, Sweden) following manufacturer instructions. The absorbance of the standard dilutions were
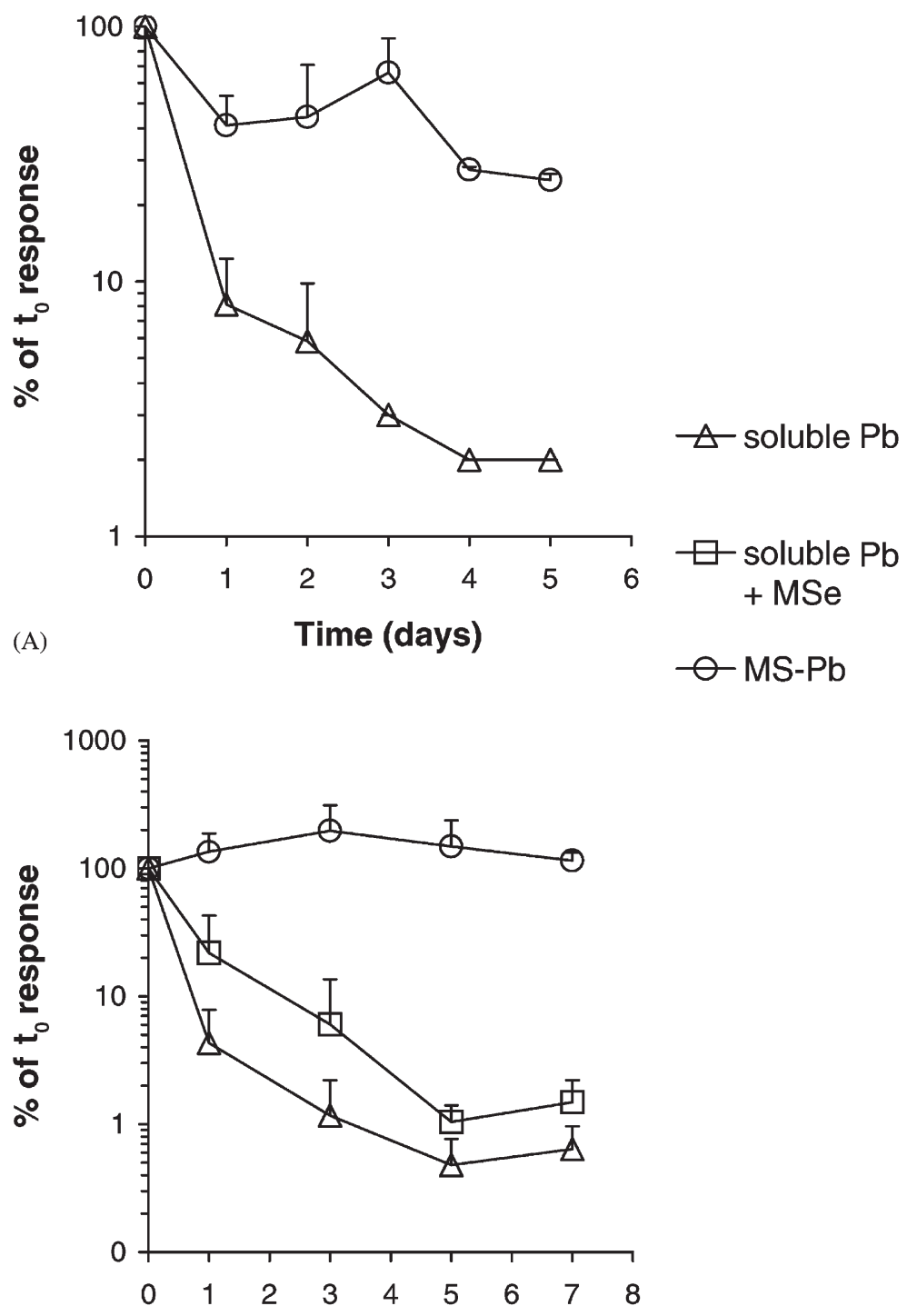

(B)

\section{Time (days)}

Fig. 1. Time course of presentation of MS-encapsulated or soluble antigen by murine macrophages. RAW cells (A) or BMM (B) were incubated overnight with a concentration of $1 \mu \mathrm{M} \mathrm{Pb}$ antigen in the form of encapsulated $\mathrm{Pb}$ (MS-Pb), soluble $\mathrm{Pb}$ (soluble $\mathrm{Pb}$ ), or for controls, empty MS with soluble $\mathrm{Pb}$ (soluble $\mathrm{Pb}+\mathrm{MSe}$ ) and without antigen \pm MSe in 24 well plates. After extensive washing, the presentation capacity of the various preparations was evaluated over time by adding $\mathrm{H}-2 \mathrm{~K}^{\mathrm{d}} / \mathrm{PbCS}_{245-253}$ specific CTLs at indicated days after antigen pulsing and collecting the supernatant for IFN- $\gamma$ measurement after $24 \mathrm{~h}$ of co-culture. Results represent the mean percentages of the responses obtained at time point zero (to) as described in Section 2. The mean percentages were determined from 3 to 9 experiments. 
fitted using a non-linear regression program and the resulting equation used to determine the international units (IU/ml) for murine IFN- $\gamma$ or $\mathrm{pg} / \mathrm{ml}$ for human IFN- $\gamma$ in supernatants. Some values of murine IFN- $\gamma$ production are expressed as percentage of the value obtained at time zero which corresponds to the time-point of addition of CTLs immediately after the washing of pulsed APC. Values represent the average of replicate supernatants obtained from duplicate or triplicate cultures.

\section{Results}

\subsection{Kinetics of antigen presentation by murine macrophages}

It is known that MS can release embedded antigen over prolonged periods of time. It has also been established that MS are efficiently taken up by macrophages. Here, we wanted to determine whether the uptake of antigen-loaded MS would result in a prolonged antigen presentation by APC to specific T cells. For this purpose, murine macrophages, from the RAW cell line and derived from bone-marrow (BMM), were incubated overnight with MS loaded with the $\mathrm{PbCS}_{245-253}$ epitope from the circumsporozoite protein of Plasmodium berghei (MS-Pb). Subsequently, after careful and extensive washing, the antigen presentation capacity of MS loaded macrophages was determined over a period of several days. To this end, $\mathrm{H}-2 \mathrm{~K}^{\mathrm{d}}$-restricted CTLs specific for $\mathrm{PbCS}_{245-253}$ were added at the time points indicated in
Fig. 1A and B. As a control, soluble antigen, empty MS or a mixture of empty MS and soluble antigen were tested in parallel. As observed, MS loaded macrophages can present the antigen at much later times than the soluble preparation. At time zero, the presentation, as measured by the level of IFN- $\gamma$ produced, was equal for the two peptide preparations.

In the case of soluble antigen, reduction of IFN- $\gamma$ production over time follows first-order kinetics with $t_{1 / 2}$ values equal to $5.4 \mathrm{~h}$ (RAW) and $12.2 \mathrm{~h}$ (BMM) which should correspond to disappearance of the peptide-MHC class I complex from the cell surface as previously described [16].

In the case of MS, the process seems to be more complex and, minimally, is composed of two different patterns. On the first day, IFN- $\gamma$ reduction was similar to that obtained with the soluble peptide. This was most likely due to the antigen burst release from MS during the first few hours, as generally observed for such systems. Subsequently, a second phase of more sustained IFN- $\gamma$ production (at least 5 days) was noted, particularly with BMM. This sustained IFN- $\gamma$ production must be ascribed to continuous supply of antigenic complexes on the APC surface, due to prolonged antigen release from ingested MS.

\subsection{Kinetics of antigen presentation by human macrophages and dendritic cells}

A third series of experiments were designed to determine the presentation capacity of human macrophages or dendritic cells (DCs), two examples of professional APC. To this end, macrophages were cultured in the presence of soluble

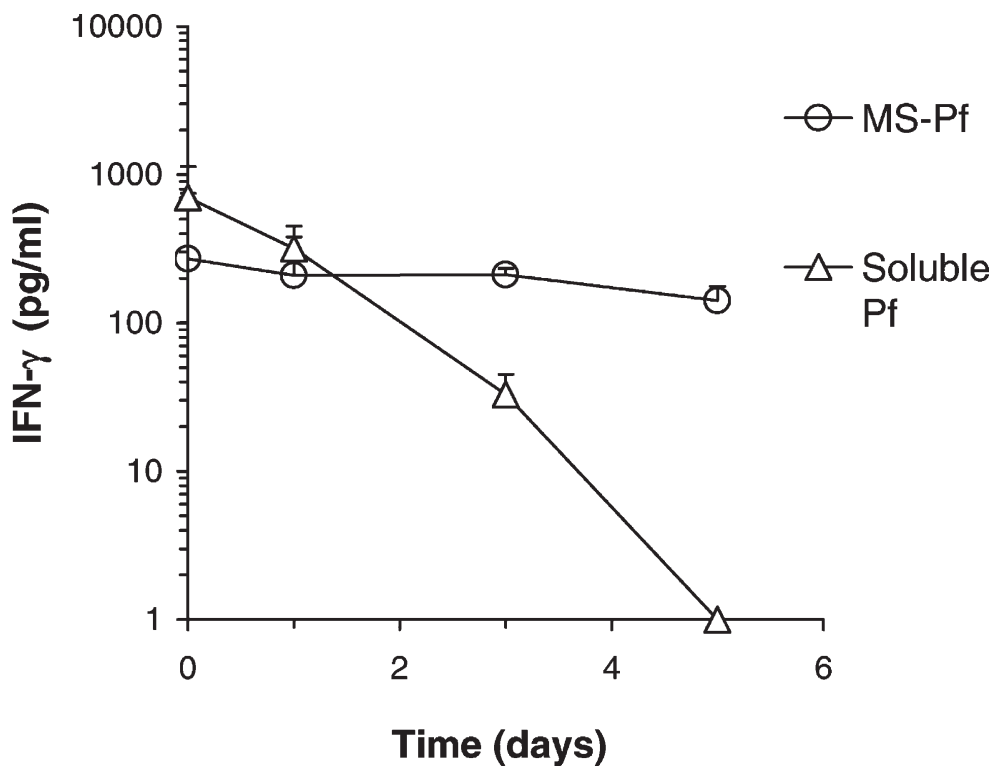

Fig. 2. Time course of presentation of MS encapsulated or soluble peptide antigen by human macrophages. Human macrophages were incubated overnight with encapsulated (MS-Pf) or soluble (soluble Pf) Pf at a concentration of $1 \mu \mathrm{M}$, empty MS (MSe) or without antigen as controls in 48 well plates. After extensive washing, the presentation capacity of the various preparations was evaluated over time by adding HLA-A0201/PfCS $334-342$ specific CTLs at the time indicated and collecting the supernatant for IFN- $\gamma$ measurement after $24 \mathrm{~h}$ of co-culture. Each value represents the mean of duplicate cultures minus the value of controls (no antigen \pm MSe). Results are representative of two experiments. 
antigen, empty MS, or antigen loaded MS and the kinetics of antigen presentation were investigated in a similar fashion as for murine macrophages. As shown in Fig. 2, while the presentation of soluble antigen by macrophages diminished with time with a half-life of $18.6 \mathrm{~h}$, the presentation of antigen loaded MS remained almost constant over 7 days.

Compared to macrophages, DCs have been described as highly superior APC with respect to activation of naïve T cells [9]. For this reason, the efficacy and duration of DC mediated presentation of MS was established. Since DCs are non-adherent, non-phagocytosed MS were separated from DCs via positive selection of cells on magnetic columns, which requires an abundant surface antigen. Among the tested antigen (CD13, CD33, CD44, and HLA DR), we obtained the best recovery of DCs using a CD44 specific antibody. CD44 is implicated in DC adhesion, and some anti-CD44 mAb can provoke DC aggregation and maturation [17]. However, the separation step using anti-CD44 labeling did not modify the presentation capacity of DCs and did not affect DC viability (results not shown). As depicted in Fig. 3, antigen presentation of two different peptides by MS loaded DCs is less constant over time than that of MS loaded macrophages but it is clearly superior to that observed with soluble antigen.
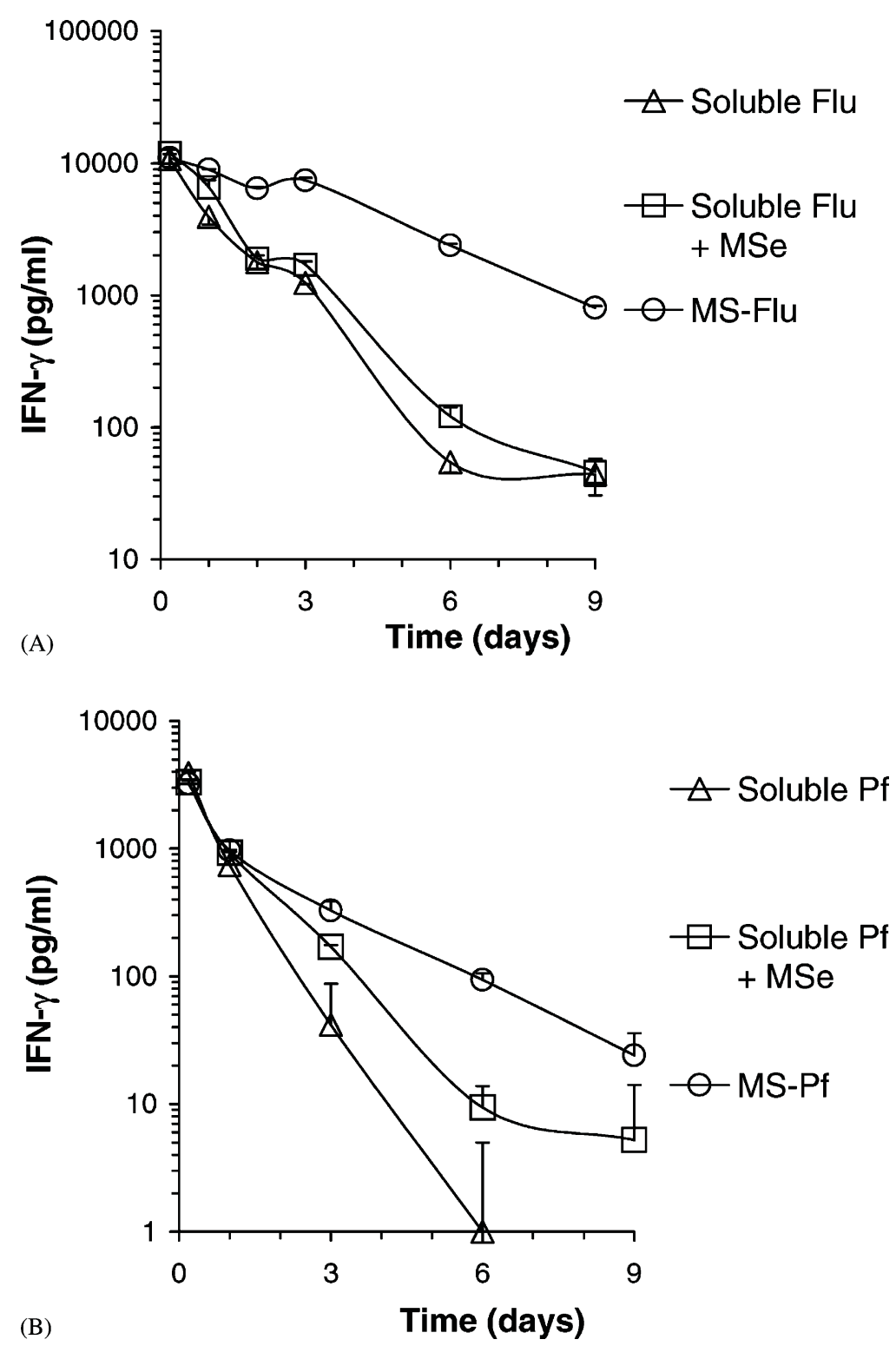

Fig. 3. Time course of presentation of MS encapsulated or soluble antigen by human DCs. Human DCs were incubated overnight with encapsulated (MS-Flu or MS-Pf) or soluble (soluble Pf or Flu) antigen $(1 \mu \mathrm{M})$, empty MS (MSe) or without antigen in 24 well plates. Free MS were removed. The presentation capacity of the various preparations was evaluated over time by adding HLA-A0201/MP 58-66 $_{\text {specific CTLs (A) and HLA-A0201/PfCS }} 334-342$ specific CTLs (B) after indicated days and collecting the supernatant for IFN- $\gamma$ measurement after $24 \mathrm{~h}$ of co-culture. Each value represents the mean of duplicate cultures minus the value of controls (no antigen $\pm \mathrm{MSe}$ ). Results are representative of two experiments. 


\section{Discussion}

Numerous studies have demonstrated that a sustained immune response can be obtained when antigens are encapsulated into PLGA/PLA microspheres ${ }^{2-8}$. The activation of a specific and prolonged immune response following a single injection of MS is probably due to the protracted release properties of biodegradable MS. Moreover, MS of a diameter below $10 \mu \mathrm{m}$ can be readily taken up by phagocytic cells which we indeed noted to occur when murine or human macrophages as well as human monocyte-derived DCs were incubated with PLGA-MS in vitro. The presentation and processing of peptides from MS-bound proteins requires the function of the proteasome $[18,19]$ suggesting that MS themselves or MS bound material reaches the cytoplasm of APC. Endocytosed PLGA-MS have been found in APC in endosomal compartments as well as in the cytoplasm, but irrespective of their localization, the pulsatile release of peptides would guarantee a prolonged supply of peptides. This is most likely the rationale for the key finding of this study, namely, that charging professional APC with peptides in an MS encapsulated form leads to a presentation of MHC class I-antigen complexes over an extended period (up to nine days). The long duration of presentation is a major advantage over external loading with soluble peptides which is associated with a short half life of antigen presentation due to the cellular turnover of MHC class I molecules and complexes on the cell surface and the lack of peptide supply from cellular stores. In the DC-based tumor vaccination trials reported to date, external loading with synthetic peptides has been performed in most cases but the clinical efficacy was low. A recent systematic analysis in the mouse model suggests that the rapid loss of externally loaded peptides from DCs contributes to this failure [20]. Loading autologous DCs with peptides in an MS encapsulated form is therefore likely to greatly improve DC-based vaccination in particular since PLGA-MS does not negatively affect survival and phenotypic maturation of DCs [21] (Y. Men, unpublished data). Since biodegradable PLGA-MS are approved and in use as clinical drug delivery systems, the loading of DCs with tumor epitopes in PLGA-MS encapsulated form deserves to be further developed and tested in tumor vaccination trials.

\section{References}

[1] Thomasin C, Corradin G, Men Y, Merkle HP, Gander B. Tetanus toxoid and synthetic malaria antigen containing poly (lactide)/poly (lactide-co-glycolide) microspheres: importance of polymer degradation and antigen release for immune response. J Control Release 1996;41:131-45.

[2] Eldridge JH, Staas JK, Meulbroek JA, Tice TR, Gilley RM. Biodegradable and biocompatible poly (DL-lactide-co-glycolide) microspheres as an adjuvant for staphylococcal enterotoxin B toxoid which enhances the level of toxin-neutralizing antibodies. Infect Immun 1991;59:2978-86.

[3] Alonso MJ, Gupta RK, Min C, Siber GR, Langer R. Biodegradable microspheres as controlled-release tetanus toxoid delivery systems. Vaccine 1994;12:299-306.

[4] Jones DH, McBride BW, Jeffery H, O’Hagan DT, Robinson A, Farrar $\mathrm{GH}$. Protection of mice from Bordetella pertussis respiratory infection using microencapsulated pertussis fimbriae. Vaccine 1995;13:675-81.

[5] Men Y, Thomasin C, Merkle HP, Gander B, Corradin G. A single administration of tetanus toxoid in biodegradable microspheres elicits $\mathrm{T}$ cell and antibody responses similar or superior to those obtained with aluminum hydroxide. Vaccine 1995;13:683-9.

[6] Men Y, Tamber H, Audran R, Gander B, Corradin G. Induction of a cytotoxic $\mathrm{T}$ lymphocyte response by immunization with a malaria specific CTL peptide based on biodegradable polymer microspheres. Vaccine 1997;15:1405-12.

[7] Partidos CD, Vohra P, Anagnostopoulou C, Jones DH, Farrar GH, Steward M W. Biodegradable microparticles as a delivery system for measles virus cytotoxic T cell epitopes. Mol Immunol 1996;33:48591.

[8] Audran R, Men Y, Johansen P, Gander B, Corradin G. Enhanced immunogenicity of microencapsulated tetanus toxoid with stabilizing agents. Pharm Res 1998;15:1111-6.

[9] Banchereau J, Schuler-Thurner B, Palucka AK, Schuler G. Dendritic cells as vectors for therapy. Cell 2001;106:271-4.

[10] Maryanski JL, Pala P, Cerottini JC, Corradin G. Synthetic peptides as antigens and competitors in recognition by $\mathrm{H}$-2- restricted cytolytic T cells specific for HLA. J Exp Med 1988;167:1391-405.

[11] Blum-Tirouvanziam U, Servis C, Habluetzel A, et al. Localization of HLA-A2.1-restricted T cell epitopes in the circumsporozoite protein of Plasmodium falciparum. J Immunol 1995;154:3922-31.

[12] Bednarek MA, Sauma SY, Gammon MC, et al. The minimum peptide epitope from the influenza virus matrix protein. Extra and intracellular loading of HLA-A2. J Immunol 1991;147:4047-53.

[13] Current Protocols in Immunology. In: Coico R, editor. Current Protocols. New York: Wiley.

[14] Sallusto F, Cella M, Danieli C, Lanzavecchia A. Dendritic cells use macropinocytosis and the mannose receptor to concentrate macromolecules in the major histocompatibility complex class II compartment: downregulation by cytokines and bacterial products. J Exp Med 1995;182:389-400.

[15] Migliorini P, Corradin G, Betz Corradin S. Macrophage $\mathrm{NO}_{2}{ }^{-}$ production as a sensitive and rapid assay for the quantitation of murine IFN- $\gamma$. J Immunol Methods 1991;139:107-14.

[16] Eberl G, Widmann C, Corradin G. The functionnal half-life of $\mathrm{H}-2 \mathrm{~K}^{\mathrm{d}}$-restricted $\mathrm{T}$ cell epitopes on living cells. Eur J Immunol 1996;26:1993-9.

[17] Haegel-Kronenberger $\mathrm{H}$, de la Salle $\mathrm{H}$, Bohbot A, Oberling F, Cazenave JP, Hanau D. Adhesive and/or signaling functions of CD44 isoforms in human dendritic cells. J Immunol 1998;161:3902-11.

[18] Men Y, Audran R, Thomasin C, et al. MHC class I- and class II-restricted processing and presentation of microencapsulated antigens. Vaccine 1999;17:1047-56.

[19] Shen Z, Reznikoff G, Dranoff G, Rock KL. Cloned dendritic cells can present exogenous antigens on both MHC class I and class II molecules. J Immunol 1997;158:2723-30.

[20] Ludewig B, McCoy K, Pericin M, et al. Rapid peptide turnover and inefficient presentation of exogenous antigen critically limit the activation of self-reactive CTL by dendritic cells. J Immunol 2001;166:3678-87.

[21] Walter E, Dreher D, Kok M, et al. Hydrophilic poly(DL-lactideco-glycolide) microspheres for the delivery of DNA to human-derived macrophages and dendritic cells. J Control Release 2001;76:14968. 